\title{
Pressure dynamics in chronic venous disease
}

\author{
T Saleem ${ }^{1}$, S Raju ${ }^{1}$ \\ 1 The RANE Center for Venous and Lymphatic Diseases, St Dominic's Memorial Hospital, 971 Lakeland Drive, Ste \\ 401, Jackson, MS 39216
}

submitted: Mar 30, 2020, accepted: Apr 18, 2020, EPub Ahead of Print: May 24, 2020

Conflict of interest: Royalty, Veniti Inc. US Patent, IVUS diagnostics. US Patent, Iliac vein stent design (Dr. Sedhadri

Raju)

DOI: 10.24019/jtavr.77 - Corresponding author:Dr. Taimur Saleem, taimur@gmail.com

(C) 2019 Fondazione Vasculab impresa sociale ONLUS. All rights reserved.

\begin{abstract}
Peripheral venous pressure is regulated by central and peripheral mechanisms. Peripheral venous hypertension is an important pathologic component of chronic venous disease and is present in about two-third of patients with chronic venous disease. It can result from reflux, obstructive lesions or high arterial inflow. The dominant influence in patients with peripheral venous hypertension appears to be obstruction rather than reflux. Reflux can be superficial or deep or both. In about $70 \%$ of patients with reflux, valvular incompetence is present in the superficial, deep and perforator systems in some combination. In an ex vivo experimental model, conduit pressure increased with smaller native or functional caliber, focal stenosis and increased post-capillary inflow. Venous pressure in the lower limb can be measured in a variety of ways: supine resting pressure, erect resting pressure and ambulatory venous pressure. These measurements
\end{abstract}

are affected by factors such as intra-abdominal pressure, intra-thoracic pressure, gravity, venoarteriolar reflux, valve reflux and venous obstruction. Venous obstruction is associated with elevated supine pressures while reflux is associated with elevated erect resting and ambulatory venous pressures. Ambulatory venous pressure reflects venous hypertension in patients with advanced venous disease. However, our investigation has shown that ambulatory venous pressure hypertension is rarely present if air plethysmography testing is negative. Consideration maybe given to the omission of the ambulatory venous pressure testing if air plethysmography testing is normal.

Keywords Peripheral venous hypertension, ambulatory venous pressure, supine venous pressure, venous obstruction, reflux, air plethysmography

\section{Introduction}

In the venous circulation, flow and pressure are important elements. However, even in chronic venous stenosis, flow is generally not severely affected because of the presence of abundant venous collaterals. However, pressure dynamics assume a more central role in venous pathology.Venous pressures are noted to be high when larger caliber veins, such as the iliac vein, occlude. The high pressure causes recruitment of additional collaterals to offset the change in venous pressure. Examples of this phenomenon include the axial transformation of the profunda femoris vein and the enlargement of the great saphenous vein in patients with occlusion of the femoropopliteal venous segment. While this compensation may restore volumetric outflow in cases with large vein occlusions, it likely remains inadequate in completely normalizing venous pressures as many patients continue to experience signs and symptoms of peripheral venous hypertension $(\mathrm{PVH})$ despite the presence of abundant collaterals noted on imaging. Collaterals are often noted to completely disappear or at least fill less robustly when a much lower resistance pathway is restored by interventions such as iliocaval venous stenting ${ }^{2}$. 
$\mathrm{PVH}$ is an important pathologic component of chronic venous disease (CVD). Venous hypertension is present in approximately two-third of patients with CVD. In CEAP classes 3 to 6 , clinical severity has shown a correlation with ambulatory venous pressure (AMVP). PVH maybe the result of valvular incompetence, obstructive venous lesions or both. In most diseased limbs, including post-thrombotic limbs, obstruction and reflux are seen to coexist ${ }^{3}$. It has been a prevailing concept that reflux is the major contributor to PVH. However, when present, iliac stenosis or obstruction actually appears to be the dominant influence on PVH, as discussed below ${ }^{1}$. It is possible that the significant stenosis or obstruction of the iliac veins hinders the occurrence of a hemodynamically

\section{Venous obstruction}

As noted above, obstruction appears to be the major contributor to $P V H$. In the setting of CVD, obstructive lesions can occur either as thrombotic, non- thrombotic or the mixed variety. Intravascular ultrasound (IVUS) is of great value in differentiating the characteristics of these lesions and guiding appropriate treatment. Thrombotic and non-thrombotic iliac vein lesions have a distinct appearance on imaging. Thrombotic lesions are segmental, irregular, multiple and diffuse often with the presence of trabeculations. On the other hand, non-thrombotic lesions are subsegmental, focal and occur at points of arterial crossovers. Mixed lesions have characteristics of both thrombotic and non-thrombotic lesions and usually occur less commonly than either subset. Use of IVUS has allowed us to appreciate the diverse array of non-thrombotic iliac vein lesions. As opposed to the classic belief, we have noted that non-thrombotic iliac veins lesions are neither exclusive to the left side, nor to young women ${ }^{7}$. The incidence of iliac vein stenosis maybe as high as $70 \%$ in the general and CVD populations ${ }^{4}$. However, it should be noted that not all iliac venous stenoses are symptomatic. Factors such as collateralization, variation in lymphatic flow and compliance changes such as venodilatation may come into

\section{Reflux}

Valvular incompetence contributes to PVH but less so than obstruction. It is likely that reflux contributes to microvascular hypertension rather than overt macrovascular hypertension. CVD patients can have reflux in the deep system, the superficial system or the perforator system. Approximately $70 \%$ patients have reflux in some combination in the superficial, deep and perforator systems. Axial reflux is reflux in the great saphenous vein to below the knee or in the femoral vein from the thigh to the popliteal vein below the knee 9 . relevant reflux. This obstruction blocks the main blood reservoir feeding the reflux, namely the iliac veins and inferior vena cava. In addition, high arterial inflow can contribute to $\mathrm{PVH}$, but this phenomenon is seen less commonly compared to reflux and obstruction ${ }^{3}$. The inter-relationship between obstruction and reflux remains unclear. Pure obstruction, in the absence of reflux, has been noted in about $30 \%$ of limbs ${ }^{4}$.

PVH is believed to cause microvascular injury. Based on animal models, chronic PVH is believed to lead to an inflammatory reaction in the venous valves over time. This, in turn, can lead to valvular dysfunction, reflux and further elevation of venous pressure upstream ${ }^{5,6}$.

play to maintain homeostasis in asymptomatic individuals, despite imaging showing iliac venous stenosis ${ }^{1}$.

Venography has inferior sensitivity compared to IVUS. In the Venogram vs IVUS for Diagnosing Iliac Vein Obstruction (VIDIO) trial, IVUS led to modification of the treatment strategies for patients with CVD. In a recent study from our center, IVUS, when used without concomitant venography, was noted to have a high diagnostic clinical yield in patients with signs and symptoms of CVD. This included patients with thrombotic as well as non-thrombotic obstructive lesions. Stenosis was noted in all of the patients $(100 \%)$ in at least one of the three veins that were examined with the IVUS (common femoral vein, external iliac vein and common iliac vein). Stenosis was also noted in all three veins in more than $50 \%$ of the patients ${ }^{8}$. In these patients, preoperative investigative studies (duplex ultrasound), history and physical examination were all noted to be suggestive of CVD. In these patients, conservative therapy had failed with the presence of significant life style limiting symptoms that were affecting their quality of life or work or both.

Treatment of the symptomatic superficial reflux involves stripping, thermal or non-thermal ablation techniques and conservative surgery while the deep system reflux can be addressed through valvular reconstruction techniques such as valvuloplasty or valve transplantation.

Pathologic perforators can be addressed through open techniques, thermal or non-thermal ablation techniques or subfascial endoscopic perforator surgery. 


\section{Quantifying saphenous reflux}

Quantification of saphenous reflux is important in CVD patients because of its impact on AMVP. Valve reflux has been seen to worsen AMVP parameters. Most venous practices currently treat pathologic reflux in the saphenous vein in the appropriate clinical setting when the saphenous vein diameter and reflux time meets criteria. We have noted that air plethysmography (APG) calf pump abnormalities are common in patients with isolated saphenous reflux and should be considered in the overall assessment of these patients when formulating an appropriate treatment plan. The calf pump has a muscle component and a venous component. The former provides contractile power while the latter provides capacitance, compliance and ejection.
Reflux volume of $\geq 30 \mathrm{ml}$ occurred mostly ( $97 \%$ of limbs) with saphenous vein size of $\geq 5.5 \mathrm{~mm}$, but also $51 \%$ of saphenous veins $>5.5 \mathrm{~mm}$ had reflux volumes $<30 \mathrm{ml}^{10}$. Reflux segment score has also been used in several studies and has been found to correlate with clinical severity. This score is determined on the basis of the presence of reflux in the number of vein segments: 1 point each is assigned to great saphenous vein, small saphenous vein, perforator, femoral vein, deep femoral vein, popliteal vein and posterior tibial vein. A score of zero indicates no reflux while a score of 7 indicates reflux in all the above mentioned segments ${ }^{11}$.

\section{Central mechanisms}

Increased arterial inflow into limb

Elevated right atrial pressure

Increased intra-abdominal pressure

Iliac vein stenosis

\section{Peripheral mechanisms}

Decreased native unstretched caliber

Decreased compliance

Focal stenosis

Venous tone

Postcapillary inflow

\author{
Arteriovenous fistula \\ Congestive heart failure \\ Morbid obesity \\ Iliac vein compression syndrome
}

\section{Clinical analogue or comment}

\author{
Maldevelopment; insufficient upscaling at venous \\ confluence \\ Decreased compliance reduces functional caliber. \\ Organized thrombus reduces luminal caliber.
}
Nearly $2 / 3^{\text {rd }}$ of general population will have silent iliac vein stenosis
Quantitative caliber effect of venous tone is unknown.
Rate of arterial inflow into the calf is increased in chronic venous disease limbs.

(adapted from Raju et $\mathrm{al}^{2}$ )

\section{Reduction of compartment pressure}

Chronic compartment syndrome (CCS) related to $\mathrm{PVH}$ from CVD is not well described in literature. The measurement of high resting compartment pressure establishes the diagnosis of CCS in the appropriate clinical context. We recently evaluated the effect of deep venous intervention (hyperdilation or new endovenous stent placement) on the compartment pressure in the posterior superficial compartment of the calf at rest in the supine position. The apparatus used for compartment pressure measurement was a simple needle manometer system using a 16-gauge needle. The posterior superficial compartment in the calf was used for pressure measurement based on tactile feel ${ }^{12}$. Based on previous reports, compartment pressure $>15 \mathrm{~mm} \mathrm{Hg}$ was considered to be indicative of venous CCS in the appropriate clinical setting. Compartment pressure was measured before intervention and then following intervention. Deep venous intervention caused a reduction in the compartment pressure of the extremity undergoing the intervention; likely by reduction of upstream venous resistance. Significant improvement 
in pain, swelling and venous clinical severity score after intervention was also noted ${ }^{12}$.

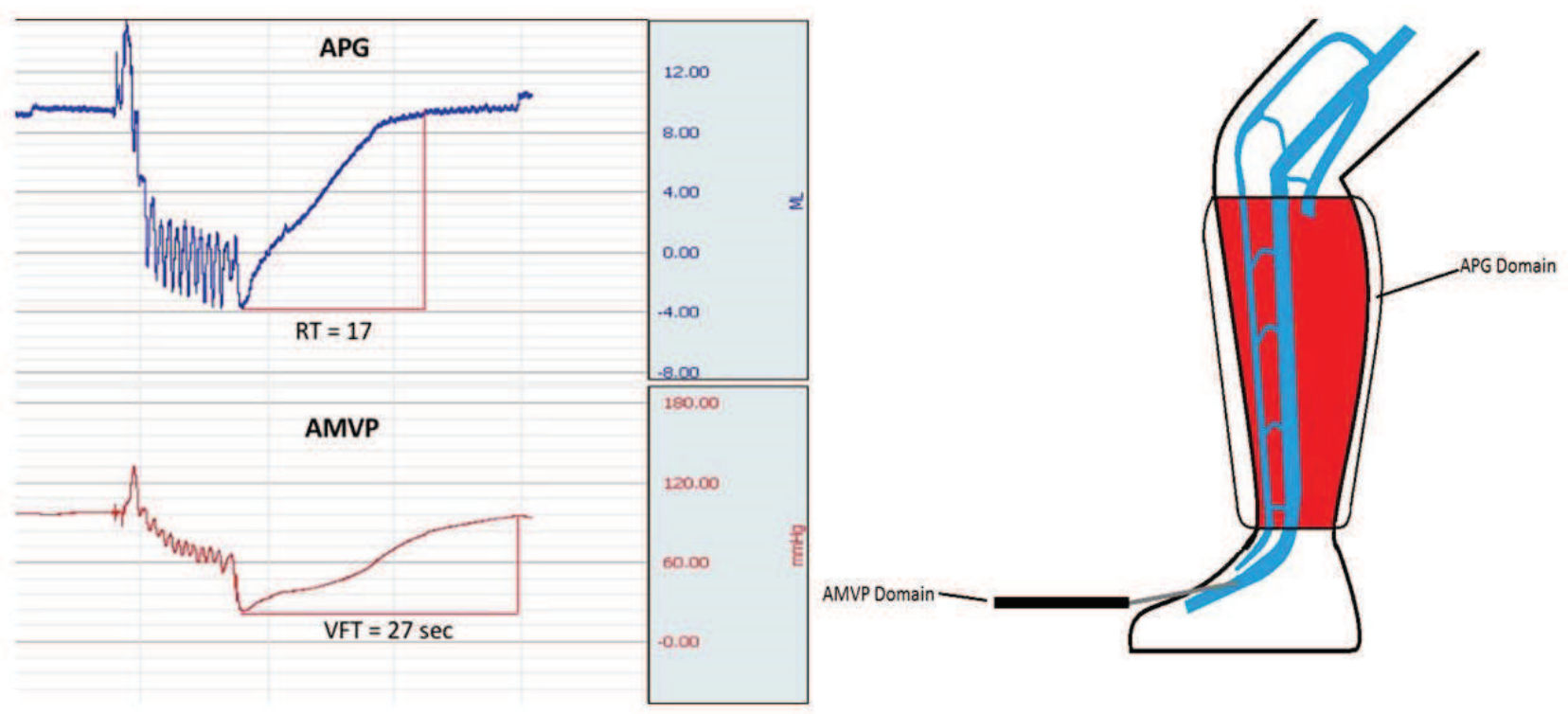

Figure 1 - APG and AMVP provide measurements in different anatomic and hemodynamic domains (adapted from Raju et $\left.a l^{4}\right)$. APG measures volume related parameters in the calf while AMVP measures pressure related parameters in the axial flow channel.

\section{Peripheral venous pressure}

Peripheral venous pressure in the lower limb is influenced by central and peripheral mechanisms (table 1, adapted from Raju et $\mathrm{al}^{2}$ ). In order to better understand peripheral factors, an ex vivo experimental venous model was constructed using penrose tubing as a venous conduit analogue. Volume-pressure curves were derived in static and dynamic modes. There was no provision in the model to simulate reflux or calf pump function. Key findings from this experimental model are as follows:

- $\quad$ 1. Decreasing compliance caused progressive reduction of conduit capacitance.

- 2. Increased postcapillary (arterial) inflow increased conduit pressure.

- $\quad 3$. For a given inflow, pressures varied inversely to conduit caliber.

\section{Measurement of venous pressure}

Venous pressure in the lower limb can be measured in a variety of ways: supine resting pressure, erect resting pressure and AMVP. These are affected by factors such as intra-abdominal pressure, intra-thoracic pressure, gravity, venoarteriolar reflux, valve reflux and venous obstruction etc $^{3}$.
- $\quad$ 4. Focal stenosis increased venous pressure if it was narrower than optimum outflow caliber.

- 5. Sideline capacitance with stagnant flow connected to the flow channel (simulating calf vein network) did not influence conduit pressure ${ }^{2}$.

Compliance, the inverse of elastance, is the ability to change volume in response to changes in transmural pressure. In our experimental model, compliance of the Penrose tubing was varied by adding layers (single layer to five ply $)^{2}$. Venous capacitance, more synonymous with venous volume, refers to the total contained volume of the vasculature over at least the physiological range of transmural pressure ${ }^{14}$. In our experimental model, sideline capacitance was increased by adding two additional Penrose tube side arms to the flow channel ${ }^{2}$.

Supine peripheral venous pressure has two components, a) a static component related to the venous fill (also referred to as "dead man pressure"), and b) an additional dynamic component generated by flow from cardiac action ${ }^{2}$. Erect venous pressure is supine pressure with the addition of a height-related gravity component. AMVP is a more global investigation of calf pump function 
as it incorporates factors like foot pump, ankle joint motion, calf muscles, capacitance and compliance ${ }^{4}$.

\section{Peripheral venous hypertension in chronic venous disease \\ Study subsets \\ CEAP classes}

We recently described the incidence and severity of PVH (supine, erect and ambulatory) in a large subset of CVD patients during a 16-year period (1999-2015) including 8868 limbs in 5792 patients $^{3}$. This was a single center retrospective observational study of data that was prospectively collected and contemporaneously entered into a time-stamped electronic medical record system. The sample was split into two subsets. Subset A $(\mathrm{n}=4132)$ had duplex ultrasound reflux status known. Subset B ( $\mathrm{n}=159)$ were taken from subset A and had IVUS-proven central obstruction (iliac vein stenosis) that was treated with endovenous stent placement. The median IVUS stenosis of limbs in subset B was $58 \%$.

\section{Pressure measurement protocol}

A needle was placed in the dorsal foot vein and was attached to a high-frequency transducer at the foot level. Supine pressure was measured in the recumbent position. Erect venous pressure was recorded in the standing position with weight bearing on the opposite limb. AMVP was recorded with 10 tiptoe exercise maneuver. AMVP drop (\% drop) and venous filling time (VFT) were calculated. On the basis of literature review, normal values were considered as: 5 to $10 \mathrm{~mm} \mathrm{Hg}$ for supine venous pressure, 80 to 100 $\mathrm{mm} \mathrm{Hg}$ for erect venous pressure, \% drop of $>50 \%$ and a VFT $>20$ seconds. A supine foot pressure $\geq 11 \mathrm{~mm} \mathrm{Hg}$ and an erect foot pressure $\geq 100 \mathrm{~mm} \mathrm{Hg}$ were considered as indicative of $\mathrm{PVH}$.

\section{Subset A}

Supine venous pressure was elevated in $70 \%$ of all diseased limbs, indicative of PVH. The highest supine venous pressure was $24 \mathrm{~mm} \mathrm{Hg}$ in this data set.

\section{AMVP and Air plethysmography}

AMVP measures pressure related parameters but is not commonly used because of its invasive nature. APG is used to measure various aspects of calf pump function - these are volume related parameters (figure 1 , adapted from Raju et $\mathrm{al}^{4}$ ). Residual volume fraction (RVF) measured via APG has been noted to have almost linear correlation with AMVP in some studies. This seems counter-intuitive as the volume pressure curve is non-linear and the two tests have different anatomic and hemodynamic
Patients in subset A were distributed across all CEAP classes, majority being in CEAP class 3 or higher. Median supine pressures were elevated $(\geq 11 \mathrm{~mm} \mathrm{Hg}$ ) in patients belonging to all CEAP clinical classes; this included CEAP clinical classes 0 to 2 . As the CEAP clinical class increased, the trend for median supine pressure, erect pressure and ambulatory pressure worsened.

\section{Reflux severity}

Erect venous pressure and AMVP showed worsening trend with increasing reflux severity. Supine venous pressures were elevated ( $\geq \mathrm{mm} \mathrm{Hg}$ ) in both reflux and noreflux groups. An association between reflux severity and elevated supine pressures was therefore not noted. Elevated erect pressures may have been seen due to the inhibition of the venoarteriolar reflex or a muted postural vasoconstrictor response in CVD limbs.

\section{Subset B}

Supine venous pressures were elevated $(\geq 11 \mathrm{~mm} \mathrm{Hg})$ with central obstruction, without a significant difference in patients with or without reflux. However, erect foot pressures worsened with reflux and central obstruction. AMVP parameters were mostly normal in iliac vein obstruction; presence of reflux worsened them ${ }^{3}$.

\section{Conclusive remarks}

Supine pressure elevation was not related to increasing reflux severity. In contrast to the prevailing concept, reflux likely has a lesser contribution to PVH. Obstruction likely has a more dominant influence on PVH. Supine venous pressures were found to be elevated in patients with central venous obstruction.

domains (figure 1$)^{4}$. RVF is determined by the residual volume plus reflux volume added after the cessation of the last calf pump contraction or activation. APG seems to provide a reproducible measure of reflux, the venous filling index 90\% (VFI90). We recently analyzed the relationship between APG and AMVP in a large subset of CVD patients spanning from 1995 to 2016 (8456 limbs in 4610 patients) ${ }^{4}$.

\section{APG parameters}


The following parameters were obtained: venous volume (VV), venous filling index (VFI90), ejection volume (EV), ejection fraction (EF), residual volume (RV), $\mathrm{RVF}$ and calf volume recovery time (RT).

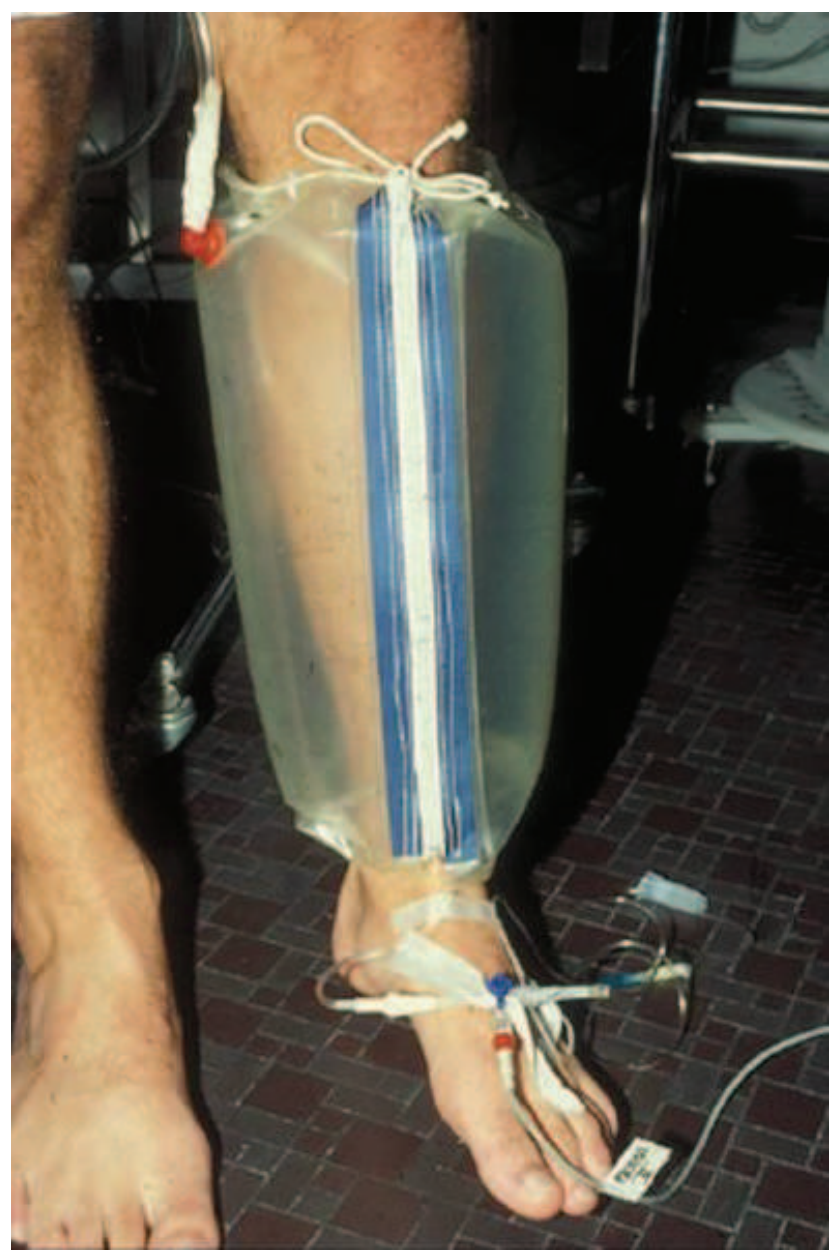

Figure 2 - Air plethysmography and ambulatory venous pressure can be performed at the same time as the tip toe movements for both tests are the same (adapted from Raju et $\left.a l^{4}\right)$

\section{AMVP parameters}

Resting pressure was obtained in the erect position with weight bearing on the opposite limb. AMVP was recorded with 10 tiptoe movements. The pressure nadir was the post-exercise pressure or AMVP (figure 2, adapted from Raju et $\mathrm{al}^{4}$ ).

\section{Calf Venous Pump}

In the calf, the superficial veins (great and small saphenous veins), deep veins (three pairs of tibial veins), muscular veins, sinusoidal veins and perforator veins are all interconnected to form a venous plexus that is influenced

\section{Results}

\section{Reflux}

$67 \%$ of the limbs had reflux in various combination (superficial, deep, perforator) while $33 \%$ of the limbs had no reflux. Steady decline was noted in the APG and AMVP parameters across various CEAP clinical classes. AMVP abnormalities were associated with CVD limbs with reflux. It was less common ( $24 \%$ only) for AMVP to be abnormal in the absence of reflux. Venn distribution of AMVP and APG abnormalities (VFI90, EF and RVF) showed that the abnormal tests between the two categories only overlapped in about $30 \%$ of the patients; this overlap increased with increasing clinical and reflux severity.

\section{Venous obstruction}

$70 \%$ limbs had obstruction with reflux while $30 \%$ had pure obstructive component. All APG parameters were noted to be normal in obstruction without reflux. VFT was normal in the group with pure obstruction. Thus, AMVP abnormalities were associated with reflux and not obstruction.

\section{Correlation between APG and AMVP}

No single APG parameter was found to be significantly helpful as a predictor for an abnormal AMVP test. Only $42 \%$ of the limbs had abnormal AMVP with a VFI90 $>3 \mathrm{~mL} / \mathrm{seconds}$. Only $7 \%$ of the limbs had an abnormal AMVP measurement in the presence of a normal APG. Therefore, consideration may be given to omitting AMVP requiring a needle stick if the APG is normal and APG may be considered a good screening test. A normal APG test result would mean either a) a non-venous cause of symptoms, or b) venous obstruction without the presence of reflux ${ }^{4}$. Both AMVP and APG tests have a higher yield with higher clinical and reflux categories.

\section{Conclusive remarks}

Traditionally, AMVP has been believed to be related to reflux. However, some authors have interpolated that AMVP could be related to obstruction. We did not find such as association in our study. AMVP abnormalities were seen in patients with reflux and not obstruction. Very few patients (7\%) had AMVP abnormalities if APG was normal.

by factors such as the calf pump ${ }^{2}$. The calf pump appears to adjust to reflux with active and passive compensation. Reflux causes an increase in the calf volume. However, the increase in VV reduces volumetric reflux into a smaller 
fraction of pump capacitance. An increase in VV causes an increase in EV. As a result, EF and RVF stay at normal or nearly normal levels (figure 3 , adapted from Raju et $\mathrm{al}^{4}$ ). Contrary to frequent citations in literature as a cause of CVD, we noted the incidence of calf pump failure to be fairly

\section{Conclusion}

The dominant influence in patients with peripheral venous hypertension is obstruction. Venous obstruction is associated with elevated supine pressures while reflux is associated with elevated erect resting and low, even in advanced clinical and reflux disease. These results are in contrast to some prevailing concepts that have postulated the incidence of calf pump failure due to fatigue or failure related to venous reflux.

ambulatory venous pressures. Ambulatory venous pressure hypertension is rarely present if air plethysmography testing is negative. The incidence of calf pump failure is fairly low, even in advanced clinical and reflux disease.

\section{Funding}

The authors received no financial support for the research, authorship and publication of this article.

\section{EV vs VV}

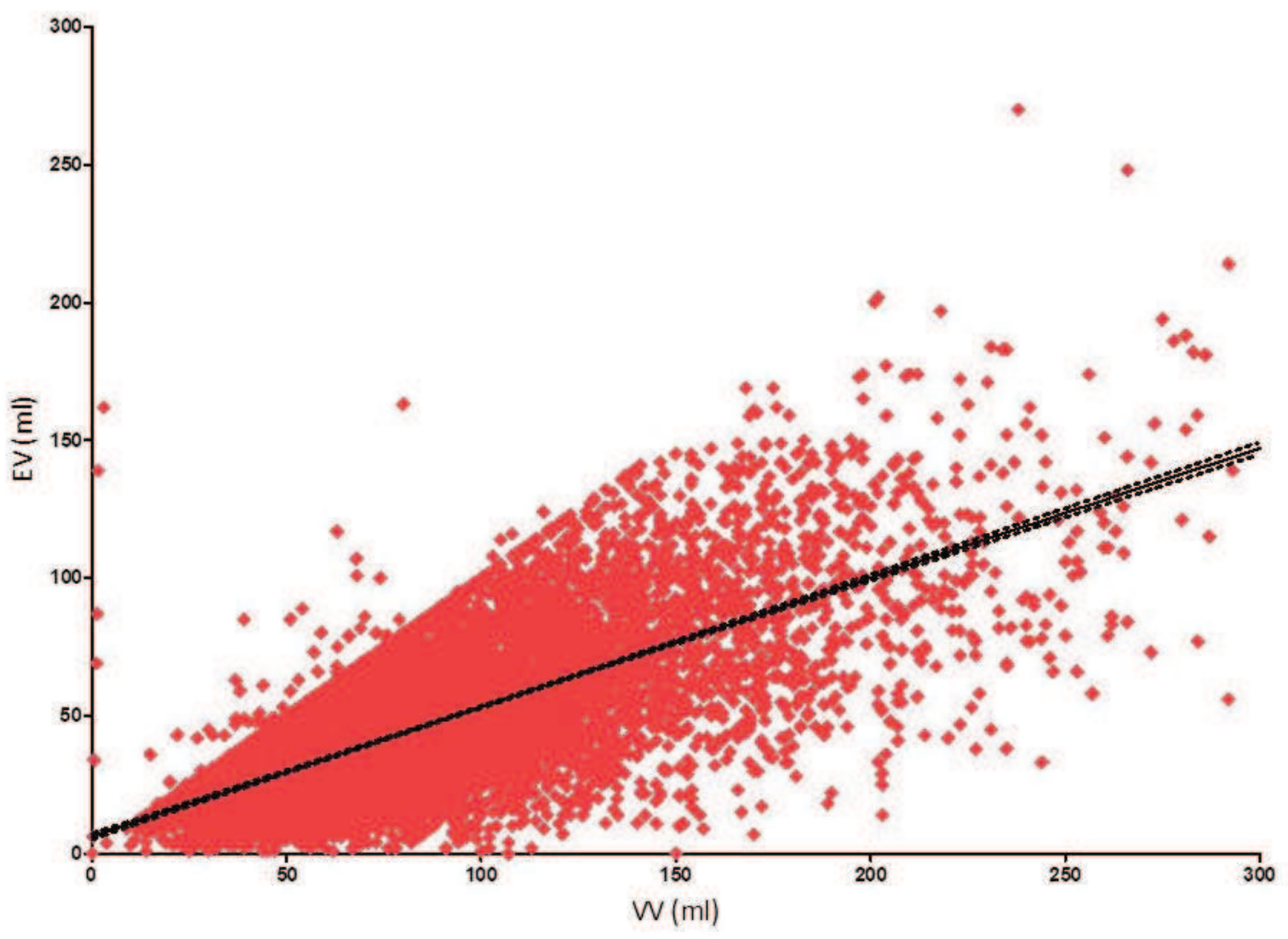

Figure 3 - Ejection volume (EV) and venous volume (VV) in 7877 limbs with chronic venous disease $(R=0.71)$. EV and $V V$ show a good linear correlation, indicating the effective compensation of the calf pump to a wide array of volumes (adapted from Raju et $\mathrm{al}^{4}$ ). Also shown are 95\% confidence bands calculated with standard errors (the confidence band is the area that has $95 \%$ chance of containing the true regression line).

\section{References}


1) Kassab G, Raju S. Grading venous stenosis is different from arterial lesions. J Vasc Surg Venous Lymphat Disord 2019;7(2):151-52.

2) Raju S, Crim W, Buck W. Factors influencing peripheral venous pressure in an experimental model. J Vasc Surg Venous Lymphat Disord. 2017 Nov;5(6):864-874.

3) Raju S, Knight A, Lamanilao L, Pace N, Jones T. Peripheral venous hypertension in chronic venous disease. J Vasc Surg Venous Lymphat Disord 2019;7(5):706-714.

4) Raju S, Knepper J, May C, Knight A, Pace N, Jayaraj A Ambulatory venous pressure, air plethysmography, and the role of calf venous pump in chronic venous disease. J Vasc Surg Venous Lymphat Disord 2019;7(3):428-40.

5) Takase S, Pascarella L, Lerond L, Bergan JJ, SchmidSchönbein GW. Venous hypertension, inflammation and valve remodeling. Eur J Vasc Endovasc Surg. 2004;28(5):484-93.

6) Pascarella L, Schmid-Schönbein GW, Bergan J. An animal model of venous hypertension: the role of inflammation in venous valve failure. J Vasc Surg. 2005;41(2):303-11.

7) Raju S, Neglen P. High prevalence of nonthrombotic iliac vein lesions in chronic venous disease: a permissive role in pathogenicity. J Vasc Surg 2006;44(1):136-144.
8) Saleem T, Knight A, Raju S. Diagnostic yield of intravascular ultrasound in patients with clinical signs and symptoms of lower extremity venous disease. J Vasc Surg Venous Lymphat Disord 2019 Dec 13: S2213-333X(19)30598-0. doi: 10.1016/j.jvsv.2019.10.021.

9) Danielsson G, Eklof B, Grandinetti A, Lurie F, Kistner RL. Deep axial reflux, an important contributor to skin changes or ulcer in chronic venous disease. J Vasc Surg 2003;38:1336-41.

10) Raju S, Ward M Jr, Jones TL. Quantifying saphenous reflux. J Vasc Surg Venous Lymphat Disord. 2015;3(1):8-17.

11) Unexpected major role for venous stenting in deep reflux disease. Raju S, Darcey R, Neglen P. J Vasc Surg. 2010;51:401-8.

12) Saleem T, Knight A, Raju S. Effect of iliofemoral-caval venous intervention on lower extremity compartment pressure in patients with chronic venous insufficiency. J Vasc Surg Venous Lymphat Disord. 2020 Feb 13:S2213-333X(20)30052-4. doi: 10.1016/ j.jvsv.2019.12.072.

13) Engelbert TL, Turnipseed WD. Chronic compartment syndrome secondary to venous hypertension: fasciectomy for symptom relief. Ann Vasc Surg 2014;28:1798.e11-4.

14) Schmitt M, Blackman DJ, Middleton GW, Cockcroft JR, Frenneaux MP. Assessment of venous capacitance. Radionuclide plethysmography: methodology and research applications. Br J Clin Pharmacol. 2002;54:565-76. 